This item was submitted to Loughborough's Research Repository by the author.

Items in Figshare are protected by copyright, with all rights reserved, unless otherwise indicated.

\title{
Computational assessment of residual formability in sheet metal forming processes for sustainable recycling
}

PLEASE CITE THE PUBLISHED VERSION

http://dx.doi.org/10.1016/j.jimecsci.2016.10.013

\section{PUBLISHER}

(C) Elsevier

\section{VERSION}

AM (Accepted Manuscript)

\section{PUBLISHER STATEMENT}

This work is made available according to the conditions of the Creative Commons Attribution-NonCommercialNoDerivatives 4.0 International (CC BY-NC-ND 4.0) licence. Full details of this licence are available at: https://creativecommons.org/licenses/by-nc-nd/4.0/

\section{LICENCE}

CC BY-NC-ND 4.0

\section{REPOSITORY RECORD}

Falsafi, Javad, Emrah Demirci, and Vadim Silberschmidt. 2016. "Computational Assessment of Residual Formability in Sheet Metal Forming Processes for Sustainable Recycling”. Loughborough University. https://hdl.handle.net/2134/22984. 


\title{
Computational Assessment of Residual Formability in Sheet Metal Forming Processes for Sustainable Recycling
}

\author{
Javad Falsafi, Emrah Demirci*, Vadim V. Silberschmidt \\ The Wolfson School of Mechanical, Electrical and Manufacturing Engineering, LE11 3TU, Loughborough, UK
}

\begin{abstract}
This paper introduces a new computational scheme addressing a problem of cold recyclability of sheet-metal products based on the assessment of their post-manufacture residual formability. Formability of sheet metals has been studied for several decades, and various techniques were suggested since a Forming Limit Diagram was first introduced in the 1960s. At the same time, cold recycling, or re-manufacturing, of sheet metals is an emerging area studied mostly empirically; in its current form, it lacks theoretical foundation. In order to address the challenge of residual formability for sheet-metal products, a reformability index is introduced in this study. The proposed method takes advantage of the latest developments in the area of evaluating multiple-path formability and introduces a quantitative reformability index for the manufactured material. This index represents possible levels of strains for deformation along different paths, based on Polar Effective Plastic Strain (PEPS). PEPS provides robustness against non-linear strain-path effects, thus making a reliable basis for such analysis. Based on residual formability, a predictive model was sought to assess a degrading effect of the flattening process. Taking advantage of extensive numerical simulation, a wide range of geometrical parameters in an unbending process, as a predominant mechanism in flattening, was studied.
\end{abstract}

The reformability index alongside prediction of degradation in flattening allows evaluation of prospective re-manufacturing. The significance of this research is its advancement towards recycling of sheet-metal products without melting them by facilitating design for sustainability. The proposed scheme also provides a subroutine friendly framework for numerical simulations.

Keywords: Remanufacturing, sheet metal, residual formability, reformability index, flattening

\footnotetext{
* Corresponding Author:

Tel: +44 (0) 1509227621 , Fax: +44 (0)1509 227648, E-mail: e.demirci@lboro.ac.uk
} 


\section{Introduction}

Environmental challenges have been considered as a serious problem across all industries, and reduction of energy and material consumption is a pressing matter for them. However, there is still much room for improvement and study. Conventional recycling of sheet-metal wastes involves melting process. High melting points of metals and additional processes required to get final products, turn it into an energy intensive process. Recycling metal waste without melting has potential for highlevel of energy saving.

In the area of sheet metals, there are a few studies that looked into the possibility of cold recycling methods and processes. Takano et al. (2008) studied the feasibility of cold recycling of sheet-metal wastes. The authors focused on deformation behaviour in incremental forming of flattened nonuniform sheet metals. They found that a forming limit of flattened sheet metals is similar to that of a sheet metal with uniform thickness. It was experimentally shown that using incremental forming; strain localisation can be almost inhibited in the flattened sheet; hence, it was concluded that cold recycling of sheet-metal wastes could be accomplished with incremental forming. Tekkaya et al. (2008) analysed remanufacturing of a contoured sheet metal part using a hydro-forming technology. They showed that using this forming process, material inhomogeneities induced by a primary forming process could be eliminated. They concluded that their technique is applicable to a re-use of sheet metal formed parts and could potentially be used e.g. to transform car bonnets for instance, into other useful shapes. The main challenge in the cold recycling/remanufacturing process is varying and inhomogeneous material behaviour as a result of the primary forming process. Therefore, a crucial requirement for re-manufacturing is the identification of residual formability in the material, and when intermediate flattening is involved, the adverse effect of flattening must be accounted for. As a matter of fact, the theoretical basis of remanufacturing has barely been addressed in the literature. The present research was an effort to bridge this gap by looking at the problem from the formability point of view.

Formability describes an extent to which a material can undergo plastic deformation without failure. A forming limit curve (FLC) shows the material ultimate formability point based on the onset of localised necking under linear loading in terms of major and minor strains. The reality of industrial applications, however, often includes substantial non-linearity in a strain history. A significant limitation of characterising formability with the FLC was highlighted experimentally by Graf and Hosford (1993). The results showed that a strain-path change resulted in deviation of the shape and location of the FLC. Hence, no unified curve in a strain space could represent the forming limit of a material. 
Alternatively, stress-based forming limits as a way to avoid a strain-path effect was proposed by Arrieux et al. (1982). They highlighted variation of the FLC in a two-stage forming process of deep drawing and flanging and proposed a stress-based forming-limit curve (SFLC). Other authors (Stoughton, 2000; Stoughton and Yoon, 2005, 2011) also constructed a forming-limit stress curve by plotting conditions for the onset of localised necking in a stress space and presented promising aspects of this approach in dealing with a non-linear strain-path effect. However, due to some shortcomings, this concept was not extensively employed. Unlike strain, stress is not easy to understand and directly measure in the experiment. Additionally, due to the decrease in the slope of a stress-strain relation, significant changes in strain occur at stress levels close to the necking limit. Thus it is difficult to visually assess or quantify the safe margin.

Stoughton and Yoon (2012) proposed an alternative approach as a remedy to the above-mentioned difficulty. They modified the idea proposed by Zeng et al. (2008) and employed effective plastic strain as a metric to evaluate formability. Effective plastic strain lies inherently in the category of stress as it is linked to the stress tensor through yield function and constitutive stress-strain relation. Eventually, they suggested a PEPS diagram and demonstrated the insensitivity of their technique to a strain path using experimental data.

With an outlook for sustainable manufacturing, the goal of this study is to introduce an index that quantifies reformability of a pre-formed material on a scale of 0 to 1 , that respectively denotes fully damaged and a healthy material. This index is strain-path-sensitive and provides an insight into the allowable formability for different strain paths.

Further, assuming that intermediate flattening is a part of a cold recycling concept, an overall approach is sought to estimate numerically a drop in reformability caused by the flattening process. The approach presented in this paper focuses on assessment with respect to geometrical parameters of an available formed material. It is believed that a sheet-metal product is more likely to be remanufactured when it was designed for it in the first place. Therefore, the presented computational scheme could contribute to design for sustainable manufacturing.

\section{Theoretical basis of reformability index}

\subsection{Polar Effective Plastic Strain diagram}

Stoughton and Yoon (2012) proposed a new approach called PEPS. They presented a review of the mapping procedure from a strain space to a stress space and pointed out that, basically, two parameters carry nonlinear paths information on. The first parameter $\beta$ is described as the ratio of the principal strain rates, while the second is effective plastic strain $\bar{\varepsilon}_{p}$, which is defined by a time integral of a function of the principal strain rates. The authors highlighted that, since the stress tensor components were identified in terms of effective plastic strain and $\alpha=\sigma_{2}^{F L C} / \sigma_{1}^{F L C}$, or indirectly in 
terms of the effective plastic strain and $\beta$, the forming limit for linear and nonlinear deformations can also be characterized as a simple limit on the accumulated effective plastic strain, $\bar{\varepsilon}_{p}$, as a function of $\beta$, or as a function of $\alpha$. In PEPS it is considered to use the effective plastic strain as one of the metrics to assess formability.

Stoughton and Yoon (2012) pointed out that although effective plastic strain was described as a type of strain, it was not directly linked to the principal or common components of the strain tensor. It is, however, uniquely linked to the stress tensor through the yield function and the stress-strain relation, and thus, falls under the category of a stress metric.

Yoshida et al. (2007) proposed the idea of an $F L C$-based on variables $\left(\bar{\varepsilon}_{p}, \alpha\right)$, and Zeng et al. (2008) presented the notion of an $F L C$-based on variables $\left(\bar{\varepsilon}_{p}, \beta\right)$. The PEPS technique which was proposed by Stoughton and Yoon (2012) is mathematically equivalent to using variables $\left(\bar{\varepsilon}_{p}, \beta\right)$, which may be more suitable for industrial engineering applications.

Therefore, to summarise the applicable equations assuming that principal strains $\varepsilon_{1}(t), \varepsilon_{2}(t)$ are parameters accounting for the strain history, the following relations are the two components of the PEPS diagram:

$$
\begin{aligned}
& \beta(t)=\frac{\dot{\varepsilon}_{2}(t)}{\dot{\varepsilon}_{1}(t)}, \\
& \bar{\varepsilon}_{p}(t)=\frac{1+r_{m}}{\sqrt{1+2 r_{m}}} \int_{0}^{t} \sqrt{\dot{\varepsilon}_{1}{ }^{2}\left(t^{\prime}\right)+\dot{\varepsilon}_{1}{ }^{2}\left(t^{\prime}\right)+\frac{2 r_{m}}{1+r_{m}} \dot{\varepsilon}_{1}\left(t^{\prime}\right) \dot{\varepsilon}_{1}\left(t^{\prime}\right)} d t^{\prime} .
\end{aligned}
$$

In order to plot the data in a polar diagram of the variable with the angle defined as the arctangent of the ratio of the principal strain rates,

$$
\theta=\tan ^{-1}\left(\frac{\dot{\varepsilon}_{2}}{\dot{\varepsilon}_{1}}\right)
$$

\subsection{Reformability index}

The nature of cold recycling necessitates a multi-stage approach to reformability. Therefore amongst available formability criteria, the least sensitive to a strain path change would be of interest. Thus, the strain-based forming limit curves are already ruled out, narrowing the options to the stress-based $F L D$ or PEPS.

The idea is to evaluate the part of material's formability exploited through forming process, and assess the residual formability. The technique proposed in this study is to calculate the area under the forming-limit curve and enclosed between the lines passing through the origin at angles $\theta=\pi / 4$ and $\theta=-\pi / 8$ (with stress triaxiality $\eta=2 / 3$ and $\eta=1 / 3$, respectively), as a quantitative measure of formability. Every stage of deformation takes off some part of this area, so the reformability index is the ratio of residual area to original area. 
Both in stress-based FLD and PEPS techniques, stages of forming correspond to a sequence of vectors towards the limit curve. Since the effective plastic strain acts as the magnitude of the strain vector, it evolves with the deformation process and; hence, changes in the area are incremental. In the stress-based FLD scheme, an evolution of strain from low to high, the stress status varies quite significantly at low strains, resulting in fast area change, while as the material get closer to the limit, the variations in the area under the limit curve becomes less and less significant. On this basis, the Polar Effective Plastic Strain technique was selected for the residual formability study.

Fig. 1 represents how the residual formability in the proposed approach could be assessed. In this figure, three forming stages with different strain path are assumed, and the respective area changes after each stage are displayed.

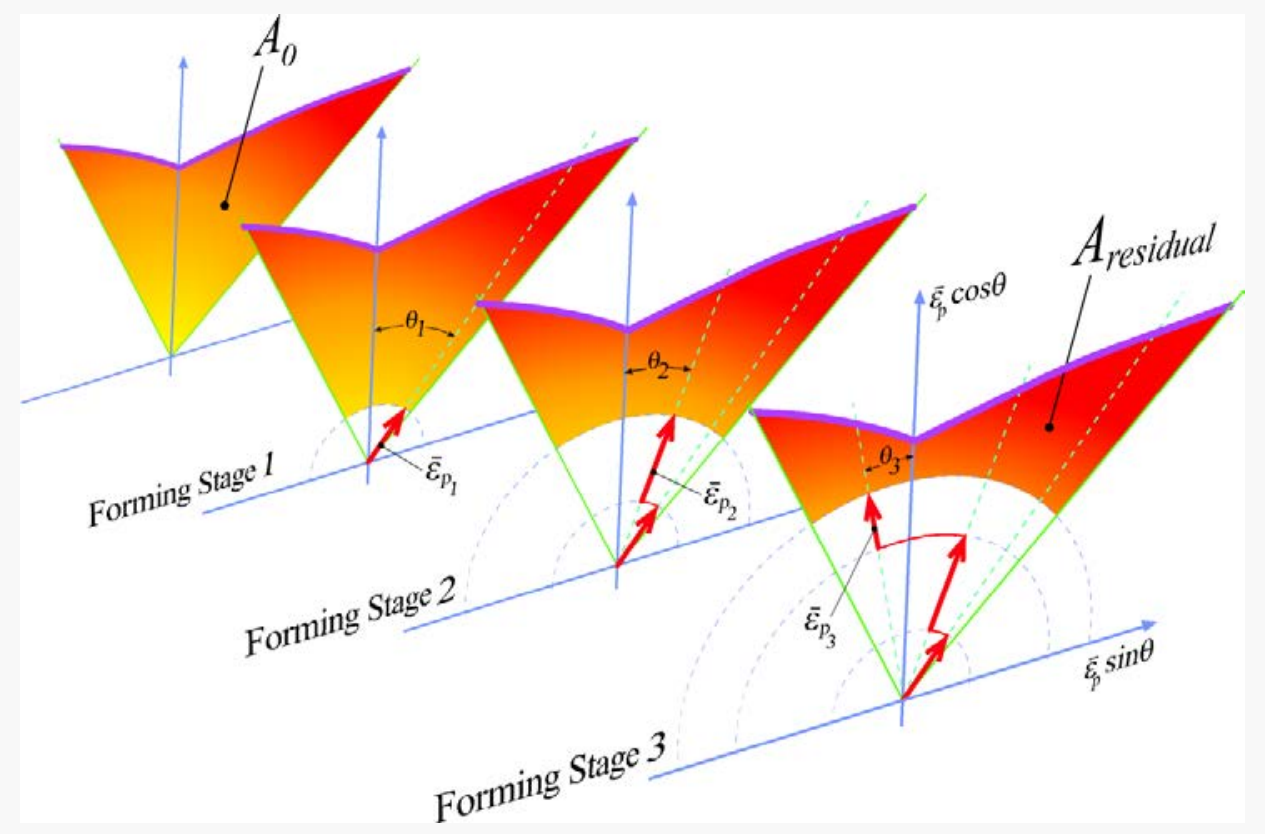

Fig. 1, The approach proposed to calculate residual formability in deformed sheet metals, uses the area under the curve as a measure of formability based on PEPS method. This area decreses with the progression of forming stages.

In the PEPS approach, the circle passing through the end point of the effective strain vector is the locus of the starting point for the successive vector. This implies that after the deformation, a circular sector would be taken off from the initial area under the limit curve. Each incremental deformation in the material enlarges this sector. The original area under the PEPS limit curve $\left(A_{0}\right)$, enclosed by $\eta=2 / 3$ and $\eta=1 / 3$ lines, minus the circular sector equals the residual area $\left(A_{r}\right)$. The reformability index $\Phi$ is then introduced as:

$$
\begin{aligned}
& \Phi=\left(\frac{A_{r}}{A_{0}}\right) \\
& A_{0}=\frac{1+r_{m}}{\sqrt{1+2 r_{m}}} \int_{-\frac{1}{2}}^{1} \sqrt{\varepsilon_{1_{F L D}}^{2}+\varepsilon_{2{ }_{F L D}}^{2}+\frac{2 r_{m}}{1+r_{m}} \varepsilon_{1_{F L D}} \varepsilon_{2}{ }_{F L D}} d \beta \\
& A_{r}=A_{0}-\frac{1+r_{m}}{\sqrt{1+2 r_{m}}} \int_{-\frac{1}{2}}^{1} \int_{0}^{t} \sqrt{\dot{\varepsilon}_{1}{ }^{2}\left(t^{\prime}\right)+\dot{\varepsilon}_{1}{ }^{2}\left(t^{\prime}\right)+\frac{2 r_{m}}{1+r_{m}} \dot{\varepsilon}_{1}\left(t^{\prime}\right) \dot{\varepsilon}_{1}\left(t^{\prime}\right)} d t^{\prime} d \beta .
\end{aligned}
$$

In a simplified form, $A_{r}$ can be expressed as: 


$$
A_{r}=A_{0}-\bar{\varepsilon}_{p}\left(3 \frac{\pi}{8}\right) .
$$

Implementing this technique using the finite element (FE) method requires either experimental data or a theoretical an FLD curve. In this study the theoretical relations to extract the FLD are used based on the local necking and diffuse necking (Hill, 1952; Swift, 1952) equations for simplicity. The conventional FLD should be projected according to the effective strain formulation into the polar domain of effective plastic strain. This is carried out according to Eq. 2 to produce the new limit diagram for assessment. The FE simulation results provide necessary information on the changes in each element of the model. Therefore, to implement the algorithm, the following calculation was performed for every element at the end of $i_{t h}$ time increment:

$$
\bar{\varepsilon}_{p_{i}}=\frac{1+r_{m}}{\sqrt{1+2 r_{m}}} \sqrt{\Delta \varepsilon_{1 i}^{2}+\Delta \varepsilon_{2 i}^{2}+\frac{2 r_{m}}{1+r_{m}} \Delta \varepsilon_{1 i} \Delta \varepsilon_{2 i}}
$$

The sequence of vectors produces a stack up of magnitudes as follows:

$$
\bar{\varepsilon}_{p}=\sum_{i=1}^{n} \bar{\varepsilon}_{p_{i}},
$$

where $n$ is the number of time increments. This allows tracking down the strain history across the material by extracting the vectors associated with the deformation of all element of the workpiece. The assembled sequence of vectors for different regions of a typical deep-drawing process is demonstrated in Fig. 2. As shown in the figure, in the polar effective-plastic-strain diagram, the limit curve remains static and as the forming process proceeds, and there is a unique strain path for each element. The forming progression entails accumulation of these incremental strain vectors, implying exploitation of initial formability to different extents in various parts of the component.

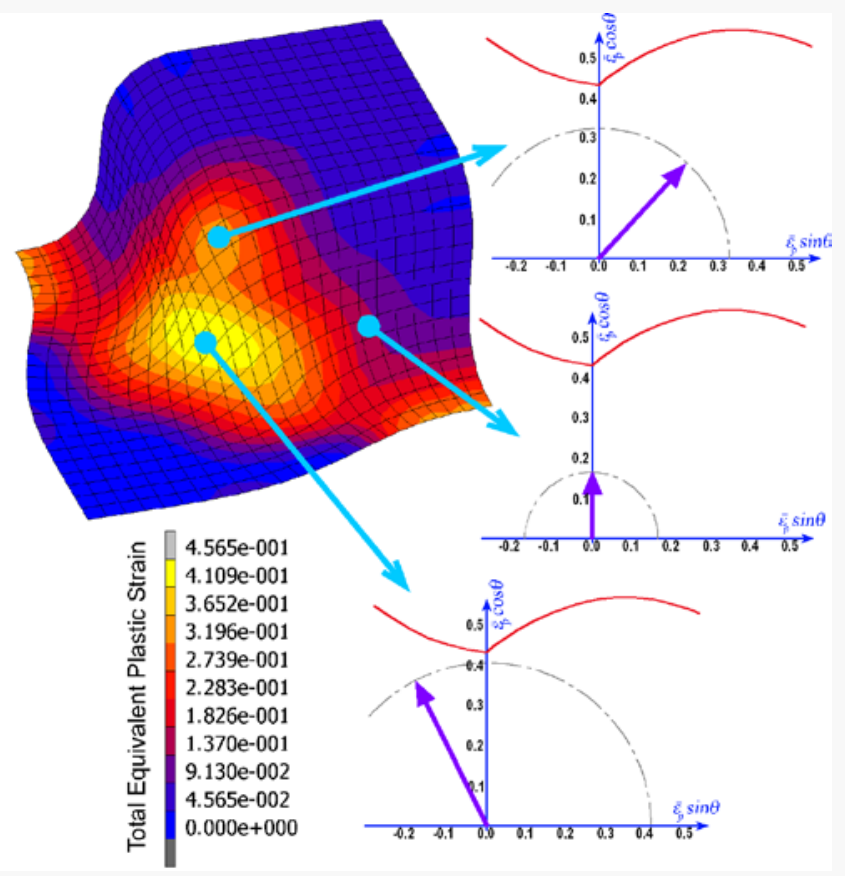

Fig. 2, Effective strain vectors in different regions of a typical quarter square deep drawing simulation in PEPS diagram. 
Two concerns need to be addressed when introducing a reformability index. First, the proposed framework delivers a quantity as the reformability index; however, direction of the subsequent strain vector is also a significant parameter in identifying the residual formability.

Second, the ratio of residual area over the original one provides a direct way for calculation of reformability index. It is still necessary to consider the effects of strain and stress gradients through the thickness, which is important for curved areas or even in flat ones that were bent and unbent while sliding over tool surfaces. Performing the formability assessment based on only the membrane values of the element's variables, could be too conservative for reliable determination of necking limits and often not conservative enough with respect to fracture.

The first concern is elaborated and dealt with in the subsection 2.3 by introducing strain path sensitive reformability index. Subsection 2.4 addresses the second concern regarding the influence of bending and unbending during the forming process is also addressed by tracking down the through thickness gradient of reformability.

\subsection{Strain-path-sensitive reformability index}

Since a single value of residual to the original area ratio does not address a clear understanding of residual formability in different strain paths, therefore the exponent $B$ is defined to modulate the reformability index to account for the strain-path effect. Therefore, $B$ is introduced as function of the strain path $=d \varepsilon_{2} / d \varepsilon_{2}$ :

$$
\begin{aligned}
& \Phi=\left(\frac{A_{r}}{A_{0}}\right)^{B}, \\
& B=B(\beta) .
\end{aligned}
$$

The benefit of introducing a non-constant exponent is to provide a convenient approach taking into account less severe necking in balanced biaxial straining, with plane strain being a critical strain condition, and the uniaxial tension falling between these two cases.

\section{Parametric study}

The ratio of a residual area to the original one conveys the residual formability and is modulated by the exponent $B$ to account for the effect of strain path on the reformability index. To have a better understanding of the proposed reformability index, the influence of these parameters is investigated. As a result of strain build-up, the effective strain becomes larger and; hence, the residual area is reduced. This incrementally reduced area, when normalised with the original area, demonstrates a linear ramp down. However, the $A_{r} / A_{0}$ ratio starts to change nonlinearly when the accumulated effective strain vector is large enough, taking off a sector crossing the limit curve. By assuming the 
PEPS limit curve obtained from a typical theoretical $F L D$, this behaviour is shown in Fig. 3 (b) as a solid line.

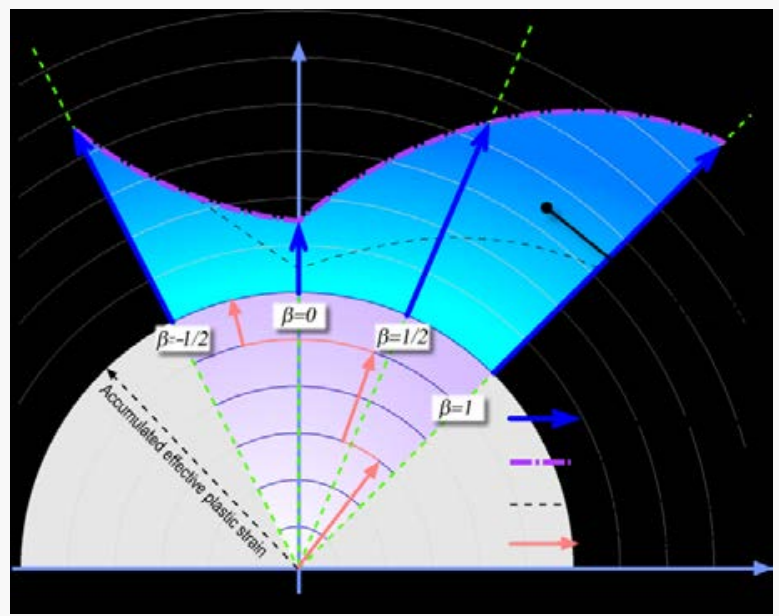

(a)

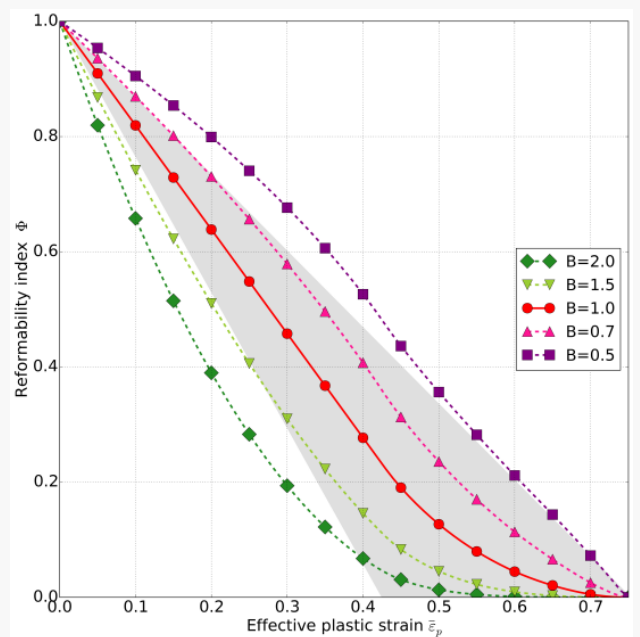

(b)

Fig. 3, Reformability index based on $\Phi=A_{r} / A_{0}$ does not provide information on residual formability for different strain path; therefore, exponent $B$ was introduced. (b) Reformability index $\Phi=$ $\left(A_{r} / A_{0}\right)^{B}$ vs. effective plastic strain for varying $B$ exponent

The exponent $B$ accounts for the strain-path direction as presented in Fig. 3. Assuming that reformability should be the ratio of a residual magnitude of the strain vectors to the original magnitudes for every strain path angle, a cloud of points was obtained at varying levels of effective strain forming the shaded region in Fig. 3. The role of exponent $B$ is to modulate the area-based reformability index so as to deliver a different value depending on strain path direction. Fig. 3 shows how the varying exponent $B$ allows for a reasonable coverage of the whole region.

The exponent $B$, is function of the strain path angle $\beta$ and can be described as a polynomial function, e.g.: 


$$
B(\beta)=C_{2} \beta^{2}+C_{1} \beta+C_{0}
$$

where $C_{0}, C_{1}$ and $C_{2}$ are the constants calculated based on the material forming limit and residual formability, as discussed below.

\section{Calculation of constants of the exponent $B$ :}

Calculation of the exponent $B$ as a quadratic function of $\beta$ requires at least three data points.

Obviously, the data points are obtained from the $F L D$, which is either an experimental curve or a theoretical curve. These data values are recommended to be obtained based on effective plastic strain equivalent to $70 \%-80 \%$ of the PEPS limit curve at $\beta=0$. Similar to Fig. 3 (a), the residual formability was calculated based on its definition at $\beta=1, \beta=0$ and $\beta=-1 / 2$. For each $\beta$ assuming, $\Phi_{\beta}$ is the vector-ratio reformability:

$$
\Phi_{\beta}=\left(\frac{A_{r}}{A_{0}}\right)^{B(\beta)},
$$

that can be linearized using $\log$ operator as:

$$
B(\beta) \log \left(\frac{A_{r}}{A_{0}}\right)=\log \left(\Phi_{\beta}\right),
$$

and, eventually:

$$
B(\beta)=\frac{\log \left(\frac{A_{r}}{A_{0}}\right)}{\log \left(\Phi_{\beta}\right)},
$$

where $A_{r} / A_{0}$ is the area-ratio reformability, independent of the strain path. This calculation gives three magnitudes of the exponent $B$, corresponding to three different values of $\beta$; this means that the constants of the quadratic equation could be extracted:

$$
\left\{\begin{array}{c}
C_{0}=B(0), \\
C_{1}+C_{2}=B(1)-B(0), \\
0.24 C_{1}-0.5 C_{2}=B(-0.5)-B(0) .
\end{array}\right.
$$

A similar procedure can also be employed to calculate four constants of the cubic equation. A sample relation $B(\beta)$ for the PEPS curve presented in Fig. 3(a) was obtained based on cubic polynomial using four data points; $\beta=1,1 / 2,0$ and $-1 / 2$; which is presented in Fig. 4.

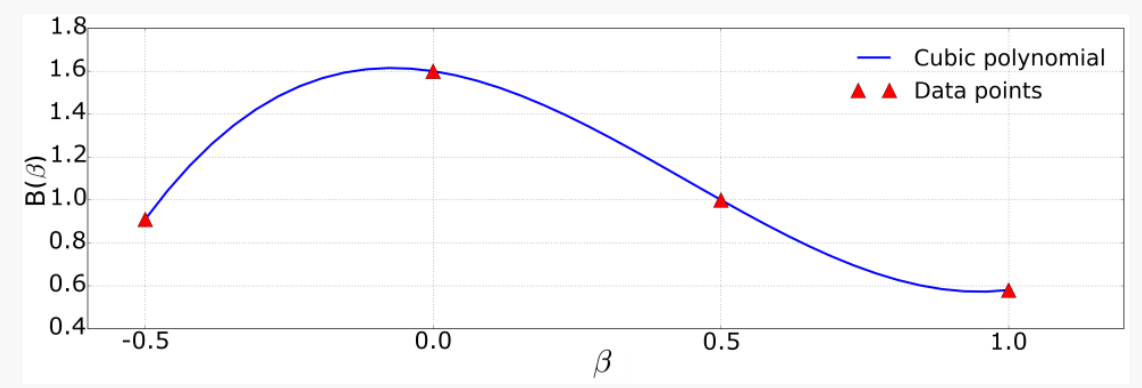

Fig. 4, Example of $B(\beta)$ for PEPS curve based on cubic polynomial. 
The reformability index based on $B(\beta)$ is presented in Fig. 5 as "predicted" $\Phi$ index. The "actual" $\Phi$ was obtained from vector ratio at every strain path angle. This figure compares the predicted reformability with the actual index and provides the error contour plot. The graphs show a good agreement; the prediction could improve with $B(\beta)$ as a higher order polynomial and also with better $F L D$. However, the maximum error of $8 \%$ is relatively small; this demonstrates the effectiveness of the exponent $B(\beta)$ in transforming the area ratio into the strain-path-dependent reformability index.

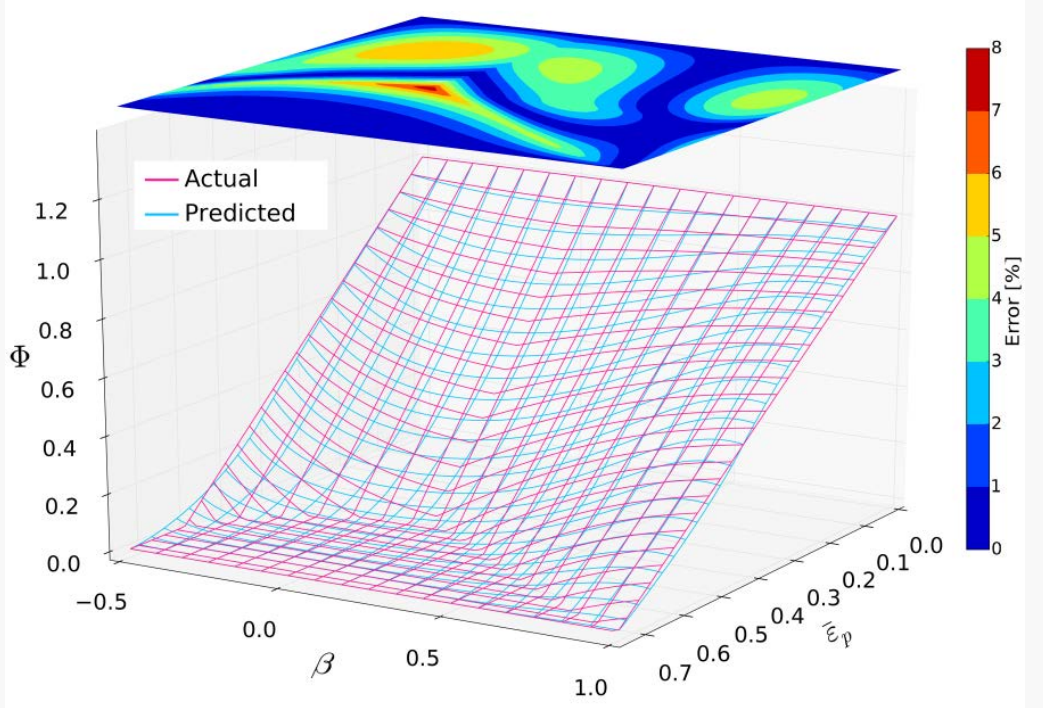

Fig. 5, Reformability index, Predicted shows the estimated reformability using $\Phi=\left(A_{r} / A_{0}\right)^{B(\beta)}$ Actual represent the actual residual formability based on the vector ratios in every strain path.

\subsection{Through thickness reformability}

As stated earlier, reformability assessment merely based on the membrane values of the element's variables could lack reliability for curved areas or even in flat ones that were bent and unbent while sliding over tool surfaces. It is easy to imagine that for the case of pure bending, for instance, there is an absence of strain in the neutral plane while tension or compression is occurring on either side towards the surface of the sheet. Given the history dependency and accumulative nature of the effective plastic strain, along with the fact that an extended level of information could be extracted from a sheet metal forming simulation when integration points through the thickness are taken into account, a potential opportunity starts to emerge.

To demonstrate this feature of the proposed idea, a simple bending of a sheet is presented with elasticplastic material model and shell type element in FEA. Fig. 6 shows the folded material, as well as strain vectors in PEPS diagram corresponding inside, neutral and outside plane of the bend. Two significant strain paths are observed on compression and tension side of the bend, while mid surface is relatively free from strain. 
Through thickness reformability calculation could be used as a mean to capture the effect of bendingunbending. This would enhance the reformability prediction and expand its applications.

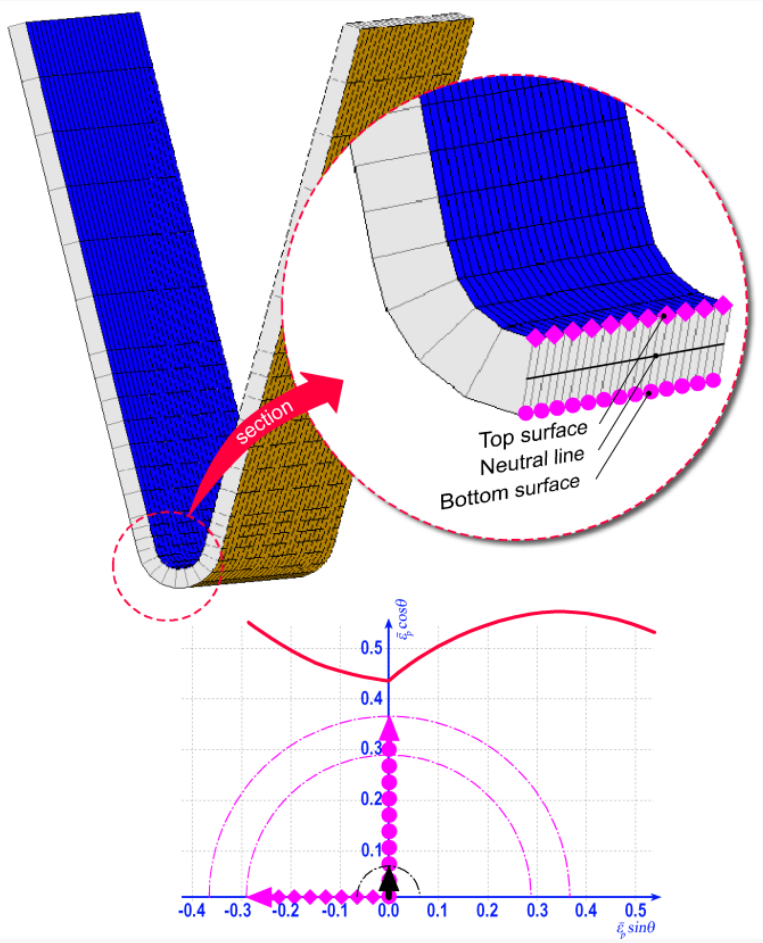

Fig. 6, While the neutral plane shows no strain change, the integration points inside and outside of the bend present a significant changes in different directions

The reformability at top, mid and bottom integration points in thickness direction are to be smeared out and provide a single representative value for reformability. Eq. 17 is proposed to account for the through thickness gradient of formability:

$$
\begin{aligned}
& \left(\frac{A_{r}}{A_{0}}\right)_{\text {rep }}=\frac{A_{r_{\text {top }}}+2 A_{r_{\text {mid }}}+A_{r_{\text {bottom }}}}{4 A_{0}} \\
& \Phi=\left(\left(\frac{A_{r}}{A_{0}}\right)_{\text {rep }}\right)^{B(\beta)}
\end{aligned}
$$

This capability of the reformability index, still required further studies and exploration. It is recommended to be used in parallel with a fracture criteria.

\section{Degradation through flattening}

The Previous sections focused on the development of a strain-path- sensitive reformability index.

Application of such a criterion falls within the domain of assessing material with regards to additional forming stages. A flattening process, however, is likely to be part of a cold recyclability scheme.

Potential degradation of material during this stage should be characterized. This section focuses on the methodology used in this research to predict the effect of flattening on levels material behaviour. The 
approach presented here is an effort towards linking the residual formability $\Phi$ of the material before and after flattening.

The presented approach employed damage as a measure of degradation; the latter was assumed to reduce a load-carrying capacity. From the microscopic point of view, material degradation within the framework of ductile damage and fracture is related to nucleation, growth and coalescence of voids in the material. Establishment of a proper material's residual formability measure requires knowledge of the first and second mechanisms and respective process parameters. Coalescence of voids is important at the final stage of ductile fracture, and since the present study deals with the material well before failure, this process can be neglected. It is also tried to connect this aspects to the geometrical parameters of the deformed material.

\section{Nucleation of voids}

According to Gurson (1977), void nucleation is a function of equivalent plastic strain. A strain-based nucleation model can reasonably describe voids nucleation in presence of positive and negative mean stresses, which is the case in bending. Therefore assuming that $\Delta$ is the degradation index of the material:

$$
\Delta_{n}=\Delta_{n}(\bar{\varepsilon}) .
$$

\section{Growth of voids}

Mean stress is known to be responsible for growth of voids (McClintock, 1968; Li et al.,2011). High mean stress accelerates the growth of voids while negative mean stress suppresses this process and delays fracture. Therefore, void growth is a function of mean stress in thickness direction:

$$
\Delta_{g}=\Delta_{g}\left(\sigma_{m}\right) .
$$

Engineering strain in thickness direction is described as:

$$
e_{x}=z / r,
$$

where $z$ is the distance from the mid-plane and $r$ is the radius of curvature at the mid-plane. As bending of sheets is widely accepted as a plane-strain process, $\varepsilon_{y}$ is negligible and $\varepsilon_{z}=-\varepsilon_{x}$. Assuming that $\varepsilon_{x} \approx e_{x}$ at small strains, it could be concluded that the most influential parameters in equivalent plastic strain, and consequently, void nucleation are radius of curvature, and sheet thickness. Therefore:

$$
\Delta_{n}=\Delta_{n}(t / r) .
$$

Assuming an accumulative nature for the equivalent plastic strain, the influence of the unbending process depends on state of the formed product in a sense that flattening process would somehow 
duplicate the existing equivalent plastic strain, with areas affected by positive mean stress experiencing negative mean stress and vice versa. As stress and stress triaxiality are linked to strain, the $t / r$ ratio could describe the void growth as well:

$$
\Delta_{g}=\Delta_{g}(t / r) .
$$

One more parameter that influences reformability of the material is the angle of bending. The higher the bending angle, the higher the strain. Therefore, eventually, degradation in reformability was assumed to be governed by the following equation:

$$
\Delta=\Delta\left(t / r, \gamma^{a}\right) .
$$

In Eq. 22, $\gamma$ is the bending angle and $a$ is the mean to modulate its influences on reformability. In order to find this function numerical simulations were employed.

\subsection{FE simulations}

In this section, a three-dimensional FE model was constructed using MSC.Marc to study the degradation of sheet material in bending-unbending process. In this investigation, both stages were simulated back-to-back to analyse the progression of degradation in the material from flat to bend, and again back to flat. The finite element model was developed based on the folding mechanism, in which the bent sheet was folded around a corner cylinder; a range of bending radii and angles were studied. In FE simulation of sheet materials, since thickness is much smaller than other dimensions, shell element was employed to improve a simulation time. The development of damage in the material was used as a measure of degradation through this process, with the Bonora damage model (Bonora, 1997) incorporated in the model. A mild steel sheet with exponential hardening law was selected as a case study for this simulation with elastoplastic material and fracture parameters tabulated in Table 1. This parameters were experimentally extracted for HD S250.

\begin{tabular}{ll} 
Table 1, Material model parameters for FE simulation \\
& HD S250 \\
\hline Material Model & Iso Elasto Plastic \\
$E$ & $200 \mathrm{GPa}$ \\
$v$ & 0.3 \\
$\sigma_{y}$ & $280 \mathrm{MPa}$ \\
$K$ & $600 \mathrm{MPa}$ \\
$n$ & 0.21
\end{tabular}

Bonora damage parameters

$\overline{\varepsilon_{t h}} \quad 0.043$




$\begin{array}{ll}\varepsilon_{f} & 0.8 \\ D_{0} & 0 \\ D_{c r} & 0.12 \\ \alpha & 0.23\end{array}$

To explore a wide range of parameters in simulations, automated parametric technique was developed to construct the entire model in MSC.Marc. Taking advantage of programmable pre-processing capabilities of a built-in py-mentat module in Marc, a large number of FE models was created according to a predefined list of parameters based on a reference simulation. A post-analyser python code using py-post module, facilitated data collection from all result files, processing and providing their assessment. A series of models were created each representing different combinations of bending angle, thickness and bending radius. These parameters covered a reasonable range of geometry and bending conditions. Table 2 shows the parameters and their corresponding values used in the analysis.

Table 2, Geometrical parameters in FE bendin-unbending simulations

\begin{tabular}{c|c|c} 
Thickness $t[\mathrm{~mm}]$ & Bend radius $r[\mathrm{~mm}]$ & Bending angle $\gamma\left[{ }^{\circ}\right]$ \\
\hline $0.5,1,1.5,2$ & $0.5,1,1.5,2$ & $30,50,70,90,120,150$
\end{tabular}

The choice of the bending severity $(r / t)$ for the thickest plate based on the tensile ductility was in accordance with available bending-limit diagrams (Hosford and Caddell, 2011). Simulations started from a flat sheet that was bent to a specified angle, followed by a release of the material to observe the spring back and the unbending stage to a fully flattened material and again released fro second spring back.

In these simulations, a bilinear thick shell element (MRAC, 2014) has been used to model the sheet with nodes in the centre plane and 13 integration points in the thickness direction.

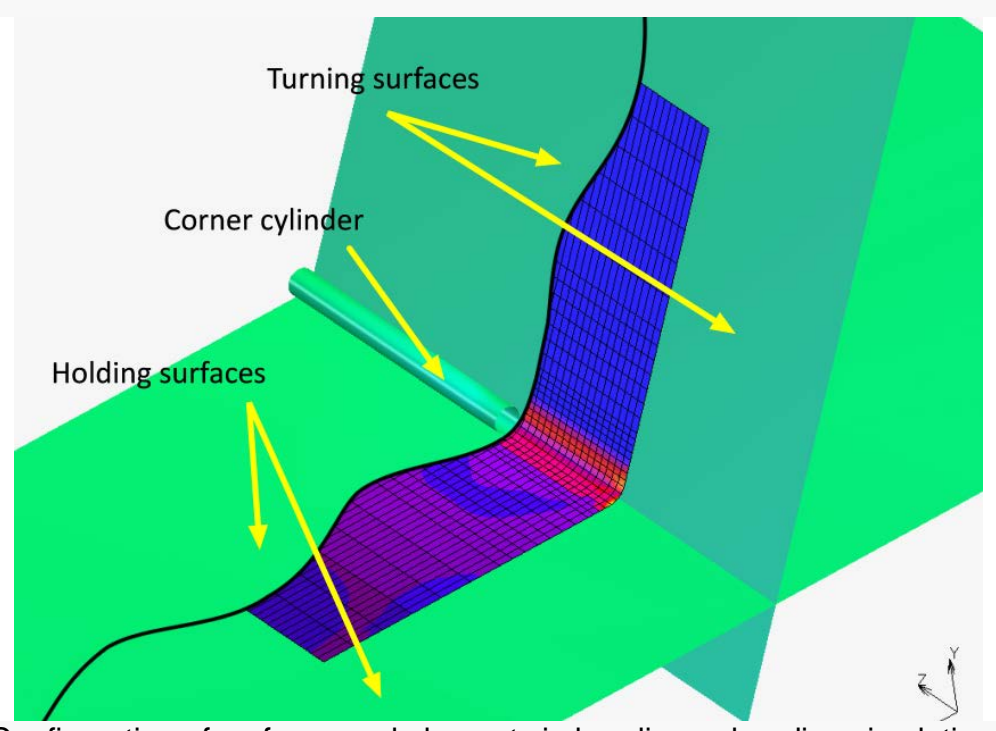

Fig. 7, Configuration of surfaces and elements in bending-unbending simulations 
The overall configuration of the developed model is depicted in Fig. 7. Two surfaces act as turning surfaces by rotating around a central axis while in contact with a folded wing of the sheet, while the holder surfaces keep the opposite wing stationary. A cylinder, sized to the bending radius, maintains the curvature of the bent zone. Mesh refinement was used in the bending zone, where a significant amount of plastic flow was expected. The python code created the models with consistency by placing ten elements along the arc length of $\pi$ radian for each radius. The applied boundary condition suppressed displacement on the edge between the holder surfaces. To describe the contact condition and interaction between the tools and the sheet, the tool surfaces were defined as rigid bodies. The material was modelled as an elasto-plastic deformable body; no friction was considered.

The key point in this study was quantifying the imposed damage (described using Bonora model) not just as a single value for an element, but for all 13 integration point as a separate quantity; this allowed assessing the extent of the damage. The reason behind the usage of the Bonora damage model was its ability to quantify the material damage in every integration point.

\subsection{Numerical analysis}

After finishing the simulations in MSC.Marc, the post processing Python code ran through the result file collected the nodal and elemental results of interest for the analysis. For each FE simulation, analysis of the nodal results was performed at two stages; first at the end of the bending followed by spring back, and second at the end of simulation after flattening and material release. To establish a basis for assessing the results, an element-wise reformability measure was calculated as:

$$
\Delta=\sum_{i=1}^{n} \frac{\left(1-D_{i}\right)}{n}
$$

where $n$ is the integration point number and $D_{n}$ is the corresponding damage. This study focused on a specific domain; Fig. 8 demonstrates the investigated region, which was in the fold zone and occupied almost three-quarters of the strip width to avoid the edge effect.

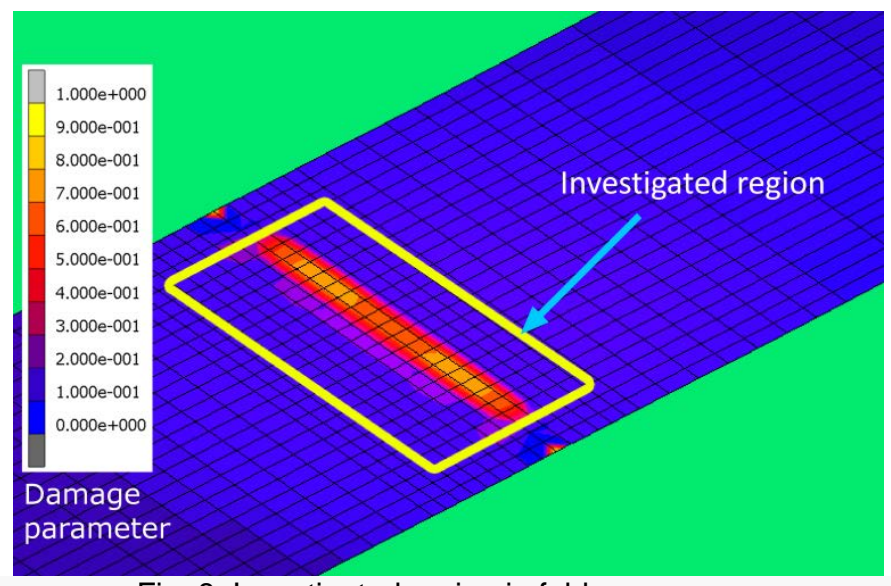

Fig. 8, Investigated region in fold zone 
It can be seen in Fig. 8 that field quantities varied in space and should be translated to representative values. Two main approaches seem to be feasible, the first one dealing with the maximum value. In this approach, regardless of the size of investigated area, the element with the highest damage represented the entire zone. The second method was based on a filtered list of elements with damage above $70 \%$ of maximum, taking the mean value of this set of elements as a representative for the region. Both methods were used to analyse the obtained results. A resultant load-bearing capacity for different levels of thickness based on the two termed "Maximum" and "Average” techniques are shown in Fig. 9. 

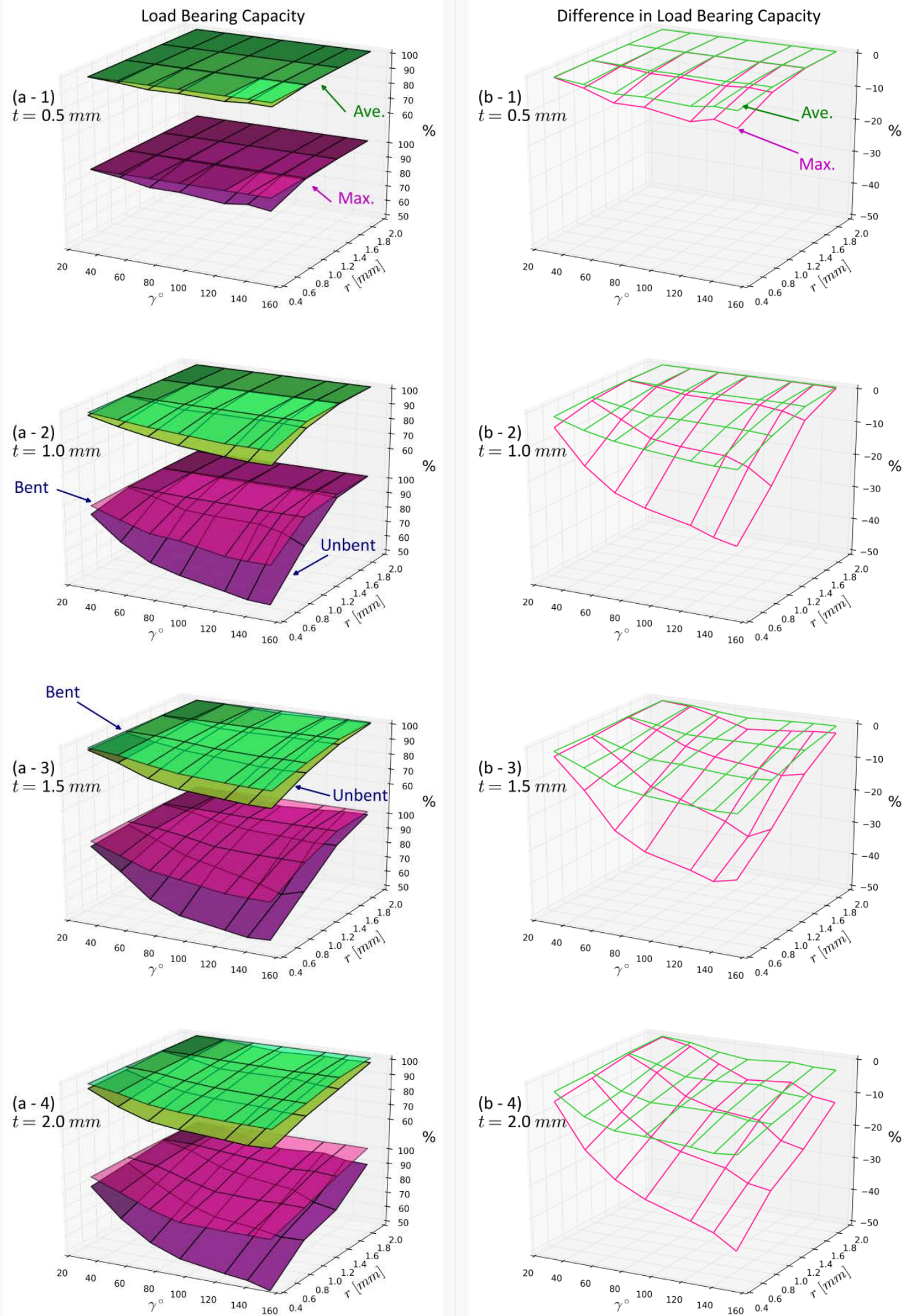

Fig. 9, (a-1 to 4) Changes in material load bearing capacity after bending and after un-bending, based on Average and Maximum, (b-1 to 4) the corresponding difference of material load bearing capacity. 
An equation was sought to predict the drop in reformability index following the flattening process. Pursuant to the discussion on influential model parameters in Section 3, all simulations were categorised based on the ratio of thickness to the radius of curvature. $t / r$ is a dimensionless parameter related to the sharpness of the bend with lower (higher) $t / r$ values corresponding to less (more) susceptibility to damage. Fig. 10 summarises all results based on the maximum drop with respect to $t / r$ and the bending angle.

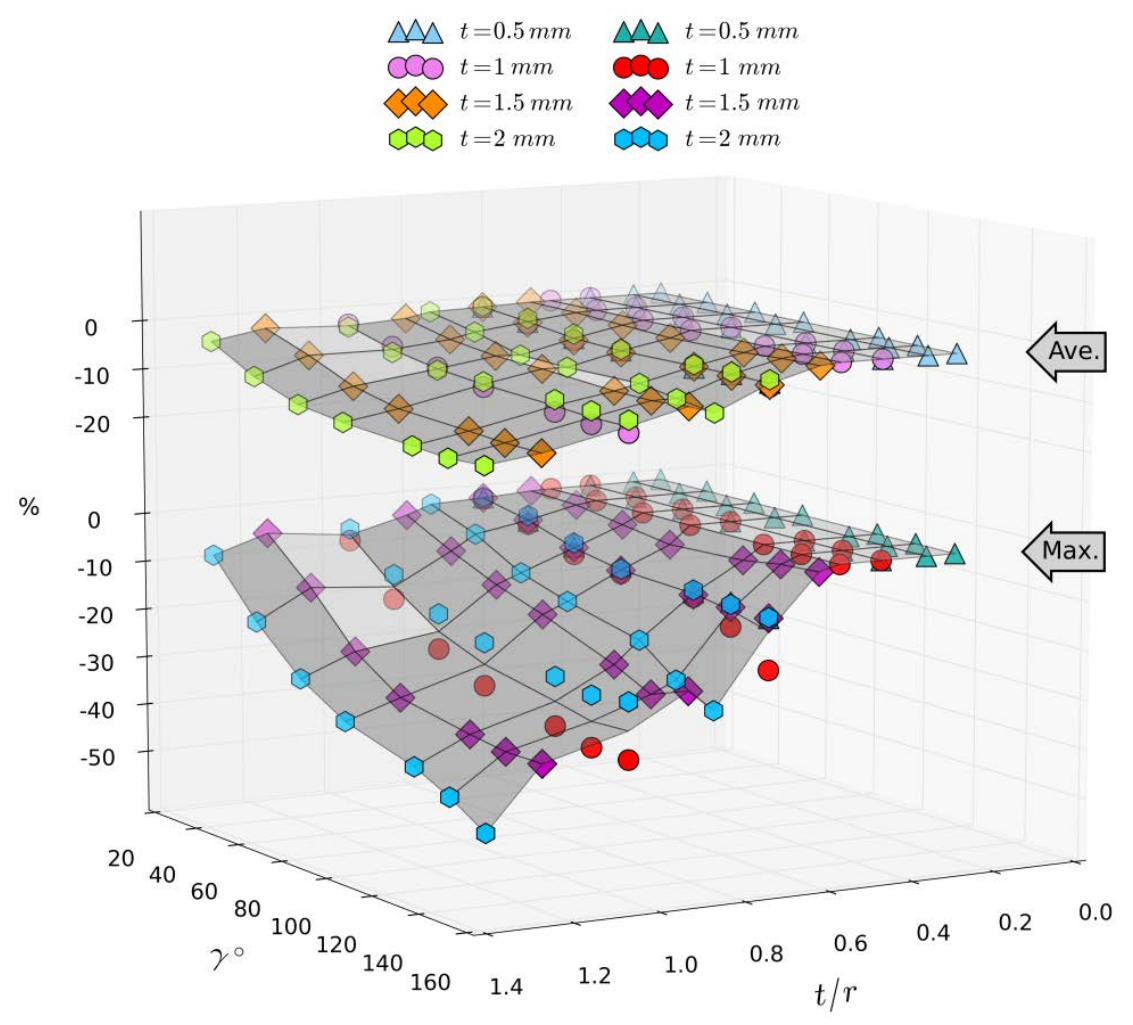

Fig. 10, Reformability change after flattening based on max and average impact technique

Apparently, the results of the "Maximum" method provided safer margins; therefore it was decided to focus on the data points based on this approach. It is seen in Fig. 10 that the load-bearing capacity decreased with the increasing bending angle. This provided an idea to combine $t / r$ and bending angle $\gamma$ in a single form. By multiplying $t / r$ with $\gamma^{a}$ (with $\gamma$ measured in radian, a dimensionless parameter), it is possible to introduce a single trend for all the cases; this is depicted in Fig. 11. The grey cloud of points is based on $t / r$, while the coloured data points represent the data based on $t /$ $r \gamma^{a}$. The figure shows that the data points tend to form a single curve and, obviously, $a$ plays an important role.

The $a$ was sought in a way to reduce the scatter in the cloud of points, leading to an optimum value of $a=0.39$ using least square curve fitting. From Fig. 11 it is visible that there is a threshold point around 0.5 in the horizontal axis, beyond which material starts to degrade, and the cloud of points 
could be represented with a declining line. Therefore, the degradation index $\Delta$ can be presented a bilinear curve. Hence, the association between geometrical parameters and degradation in flattening, presented by Eq. 22 has the following form:

$$
\Delta(x)=\left\{\begin{array}{ll}
1 & x<0.5 \\
1+(-0.32 x+0.18) & x \geq 0.5
\end{array} \text { where } x=\frac{t}{r} \gamma^{a}\right.
$$

As $\Delta$ described the change in reformability index after flattening for the modelled material in this research, the post-flattening residual formability is:

$$
\Phi_{\text {post-flattening }}=\Phi_{\text {pre-flattening }} \cdot \Delta
$$

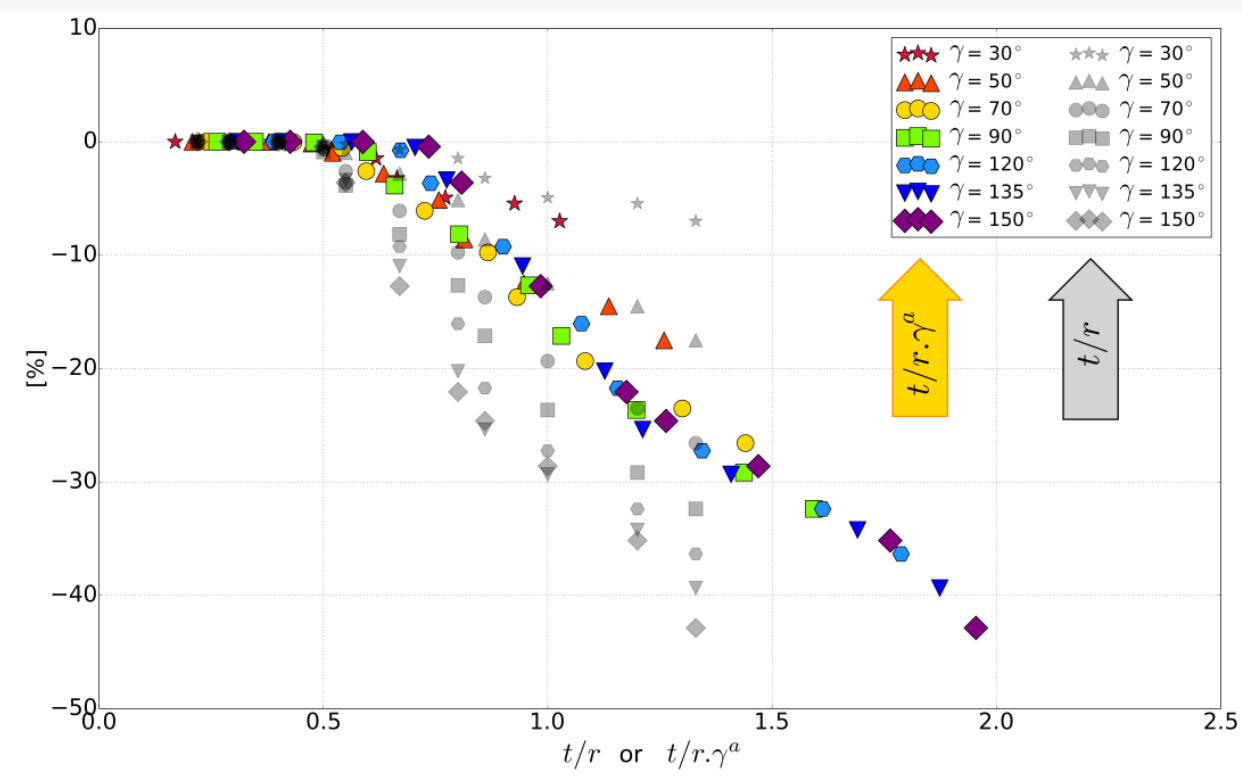

Fig. 11, Change in load-bearing capacity for various $t / r$ and $\frac{t}{r} \gamma^{a}$

\subsection{Verification}

A comparison was made to verify the developed model and experimental results presented by Falsafi and Demirci (2016) for the formability assessments after unbending the fold zones of a cold roll formed profile. Their experiment was carried out on the same material used in this study, and based on the reported change in formability and the geometrical parameters in their profile, three points are extracted for verification ( see Table 3 ). The analytical prediction of the drop in formability after flattening is shown as asolid line in Fig. 12, together with the experimental points. Considering the number of experiments and the limited range of parameters, clearly further tests are required, however, it is visible that the analithical model have provided a close prediction of available experimental results. 
Table 3, Experimental results for residual formability after bending and flattening presented by Falsafi and Demirci (2016)

\begin{tabular}{lcccccc} 
Sample & \multicolumn{2}{c}{ Formability } & & Bending angle & $t / r \gamma^{a}$ & $\Delta$ \\
\cline { 2 - 3 } & Bent & Flattened & & & \\
\hline 1 & 0.90 & 0.80 & 70 & 0.83 & 88.88 \\
2 & 0.89 & 0.79 & & 90 & 0.91 & 88.76 \\
3 & 0.90 & 0.78 & 100 & 0.95 & 86.66
\end{tabular}

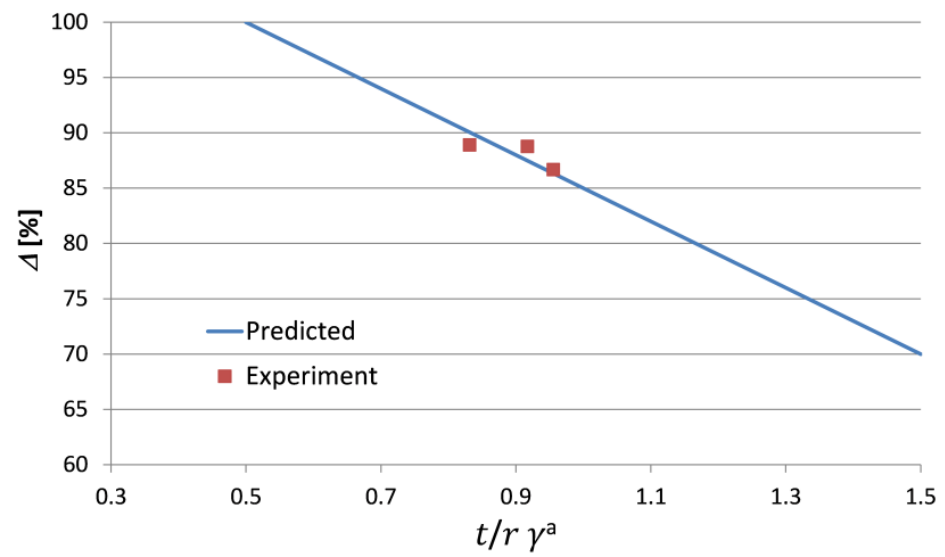

Fig. 12, Experimental and predicted results for reformability change after flattening.

\section{Conclusions}

Two goals were pursued in this paper. The first was to introduce a reformability index that quantifies the reformability of the material in deformation process with arbitrary strain path on a scale from 0 to 1 , corresponding to a fully damaged and a healthy material. This scheme was proposed based on the newly introduced PEPS which is independent of strain-path changes. This important attribute helped to develop a reformability criterion, which provides a strain-path-sensitive index. The methodology was established and formulated in this paper, together with a way to obtain the relevant constants of the developed model.

The second part of this study was an effort to quantify the process of material degradation due to the flattening process. An extensive numerical study was conducted to predict the material degradation in flattening of bent materials. A series of 3D simulations of folding, followed by unfolding of the flat sheet material were generated using Python programming for MSC.Marc. Sets of different thicknesses, bending radii and angles were chosen to provide enough information for the 
subsequent data analysis. For this analysis, another Python code was developed that could run through all the result files and extract the intended entities at specific time steps along with sorting and storing the data for analysis in a predefined order.

The analysis was then carried out by assessing the damage occurred in the material as an indication of its degradation. By assessing this attribute in the material at the end of bending as well as after unbending, a drop in load-bearing capacity for all simulations was quantitated. These data were analysed to understand the affecting parameters and presented in different forms.

It was intended to obtain a model for characterization of the additional damage resulted from unbending based on the geometrical parameters of the process. This model was eventually extracted and presented in this paper. The generic form of the model is constructed with consideration of damage accumulation induced by nucleation and growth of voids. These processes were associated with the geometrical parameters; thickness, curvature and bending angle. The presented model was compared with the experimental results. The comparison showed good agreement, demonstrating that the model could reasonably present a close estimation of the effect of flattening on formability. The presented model is suitable to be incorporated in numerical platforms.

\section{References}

Arrioux, R., Bedrin, C. and Boivin, M., 1982, May. Determination of an intrinsic forming limit stress diagram for isotropic metal sheets. InInternational Deep Drawing Research Group, Proceedings of the 12th Biennial Congress IDDRG (pp. 61-71).

Bonora, N., 1997. A nonlinear CDM model for ductile failure. Engineering fracture mechanics, 58(1), pp.11-28.

Falsafi, J. and Demirci, E., 2016. Micro-indentation based study on steel sheet degradation through forming and flattening: toward a predictive model to assess cold recyclability. Materials \& Design, 109, pp.456-465.

Graf, A. and Hosford, W., 1993. Effect of changing strain paths on Forming Limit Diagrams of Al 2008-T4. Metallurgical transactions A, 24(11), pp.2503-2512.

Gurson, A.L., 1977. Continuum theory of ductile rupture by void nucleation and growth: Part I-Yield criteria and flow rules for porous ductile media.Journal of engineering materials and technology, 99(1), pp.2-15.

Hill, R.T., 1952. On discontinuous plastic states, with special reference to localized necking in thin sheets. Journal of the Mechanics and Physics of Solids, 1(1), pp.19-30.

Hosford, W.F. and Caddell, R.M., 2011. Metal forming: mechanics and metallurgy. Cambridge University Press.

Li, H., Fu, M.W., Lu, J. and Yang, H., 2011. Ductile fracture: experiments and computations. International journal of plasticity, 27(2), pp.147-180.

Marc, M.S.C., 2014. Volume B: Element Library. MSC. Software Corporation.

McClintock, F.A., 1968. A criterion for ductile fracture by the growth of holes.Journal of applied mechanics, 35(2), pp.363-371.

Stoughton, T.B. and Yoon, J.W., 2011. A new approach for failure criterion for sheet metals. International Journal of Plasticity, 27(3), pp.440-459.

Stoughton, T.B. and Yoon, J.W., 2012. Path independent forming limits in strain and stress spaces. International Journal of Solids and Structures,49(25), pp.3616-3625. 
Stoughton, T.B. and Yoon, J.W., 2005. Sheet metal formability analysis for anisotropic materials under non-proportional loading. International journal of mechanical sciences, 47(12), pp.1972-2002.

Stoughton, T.B., 2000. A general forming limit criterion for sheet metal forming. International Journal of Mechanical Sciences, 42(1), pp.1-27.

Swift, H., 1952. Plastic instability under plane stress. Journal of the Mechanics and Physics of Solids, 1(1), pp.1-18.

Takano, H., Kitazawa, K. and Goto, T., 2008. Incremental forming of nonuniform sheet metal: Possibility of cold recycling process of sheet metal waste. International Journal of Machine Tools and Manufacture, 48(3), pp.477-482.

Tekkaya A. E., Franzen V., Trompeter M., "Wiederverwertungsstrategien für Blechformteile", 15. SFU (2008), 187-196.

Yoshida, K., Kuwabara, T. and Kuroda, M., 2007. Path-dependence of the forming limit stresses in a sheet metal. International Journal of Plasticity,23(3), pp.361-384.

Zeng, D., Chappuis, L., Xia, Z.C. and Zhu, X., 2008. A path independent forming limit criterion for sheet metal forming simulations. SAE International Journal of Materials \& Manufacturing, 1(1), pp.809-817. 